REVIEWS

\title{
Research progress of SALL4 gene in human malignant tumor
}

\author{
Jue Wang, Huijie Zhang* \\ Department of Oncology, The Third Affiliated Hospital of Inner Mongolia Medical University, Baotou, Inner Mongolia, China
}

Received: January 23, 2018

DOI: $10.14725 /$ dcc.v5n1p32
Accepted: March 4, 2018

Online Published: March 10, 2018

URL: http://dx.doi.org/10.14725/dcc.v5n1p32

\begin{abstract}
At present, malignant tumor is a serious threat to human health. Its pathogenesis is complicated and caused by the interaction of various carcinogenic factors in human body. Human sal-like protein 4 (SALL4) is a newly discovered gene. It can play an important role in the function of embryonic stem cells since it is with protein transcription factor of $\mathrm{C} 2 \mathrm{H} 2$ zinc fingers. Studies have found that mutations of the gene often lead to the occurrence of malignant tumors. This article reviews the molecular mechanism of SALL4 in the occurrence and development of malignant tumors and its role in the diagnosis and treatment of malignant tumors.
\end{abstract}

Key Words: Proto-oncogene, SALL4, Tumor

Due to the continuous development of economy, serious environmental pollution and the accelerated pace of population aging of our country, the incidence and mortality of cancer in China are rising rapidly. At present, the incidence and death toll of liver cancer, stomach cancer, lung cancer and esophageal cancer in China rank first in the world. ${ }^{[1]}$ The occurrence and development of malignant tumor is a very complicated process. It is not only related to individual genetics, bad living habits, and tumor prevention and screening, but also related to the imbalance of proto-oncogene and anti-oncogene, gene mutation or inactivation, the replication and blocking of apoptosis of malignant tumor and so forth. The expression quantity of human sal-like protein 4 (SALL4) decreases gradually with the maturation of tissues and organs. However, the quantity of SALL4 gene is highly expressed in malignant tumors. Therefore, the exploration of SALL4 gene will reveal the occurrence mechanism of tumor. This article mainly reviews molecular mechanism of SALL4 gene, and its potential on cancer diagnosis and treatment.

\section{Biological characteristics of SALL4}

SALL4 is a newly discovered proto-oncogene with two subtypes: SALL4A and SALL4B. In early embryonic stage, SALL4A and SALL4B may come from homodimer or the different combinations of homodimer with different DNA binding sites. ${ }^{[2]}$ The expression of SALL4 can be found as early as in stage 4 embryo cells. Destruction of SALL4 gene will lead to embryo death. Low expression of SALL4 in early stage may lead to deformity of embryonic development. This suggests that SALL4 is essential for early embryonic development. In embryonic cells, self-renewal of SALL4 is important. The promoter of SALL4 is STAT3 binding region, which has the ability to activate cellular selfrenewal and the functional regulation of embryonic stem cells. ${ }^{[3]}$ At the same time, STAT3 transduction pathway

*Correspondence: Huijie Zhang; E-mail: zhanghuijie1210@163.com; Address: Department of Oncology, The Third Affiliated Hospital of Inner Mongolia Medical University, Baotou, Inner Mongolia, China. 
is closely related to the gene expression of tumor cells. It is reported that Sonic Hedgehog signaling pathway plays a significant role in the process of organ differentiation. ${ }^{[4]}$ SALL4 can regulate the Sonic Hedgehog signaling pathway and block the differentiation of embryonic stem cells. In general, overexpression of SALL4 is involved in tumor genesis and progression. At the same time, SALL4 regulates many different target genes in different types of cancer cells.

\section{SALL4 and biological behavior of malig- nant tumors}

\subsection{SALL4 gene and cell transformation}

In human body, SALL4 gene mutation is an autosomal dominant disease often involving multiple organ defects, which can lead to Duane Radial Ray Syndrome (Okihiro Syndrome). ${ }^{[5]}$ SALL4 gene expression also can be found in acute myeloblastic leukemia, chronic myelocytic leukemia, breast cancer, lung cancer, gastric cancer, colorectal cancer, liver cancer, endometrial carcinoma and other cancers. ${ }^{[6,7]}$ In the normal human blood system, SALL4 is highly expressed in the positive hematopoietic stem cells/ancestral cells of CD34. But the expression decreases gradually in the course of hematopoietic cell maturation. Mature myeloid cells do not express SALL4 gene at all. Correspondingly, the expression of SALL4 is abnormally high in human acute myeloid leukemia (AML). In order to prove whether abnormal expression of SALL4 factor resulted in AML, Ma et al. ${ }^{[8]}$ studied on mouse model with SALL4 and found that overexpression of SALL4 led to abnormal proliferation of bone marrow with hematopoietic cell defect and the number of erythrocytes, granulocytes and platelets in peripheral blood gradually decreased at the same time. Typical myelodysplastic syndrome (MDS) would occur in mice 6 to 8 months after birth, and about half of the mice end up with acute leukemia. After being transferred SALL4 factor with AML evoking function, the nude mice would eventually develop into acute myeloid leukemia. It means that the factor is with transferability. The study suggests that the signaling pathway for leukemia caused by SALL4 factor has been identified. SALL4 factor binds to $\beta$-catenin, thereby activating $\mathrm{Wnt} / \beta$-catenin signaling pathway. From the mouse model we can see that overexpression of SALL4 can significantly increase the target gene level of the downstream c-myc and cyclin D1 of Wnt/catenin signaling pathways. In addition, studies have shown that in the course of leukaemia, Bmi-1 gene is regulated by SALL4. ${ }^{[9]}$ As a recognized carcinogen, Bmi-1 plays an important role in regulating stem cell function and occurrence of leukaemia. During the process from normal hematopoietic cells to myelodysplastic syndrome or acute myeloid leukemia, the overexpression of SALL4 factor increased the expression of Bmi-1 in mice.

\subsection{SALL4 gene and cell proliferation and apoptosis}

SALL4 gene is the key factor of cancer cell proliferation and apoptosis. Removing SALL4 gene can make leukemia cell apoptosis and growth arrest. ${ }^{[10]}$ In addition, downregulation of SALL4 can significantly reduce the carcinogenicity of leukemia cells. In human leukemic cells, combination of chromatin immunoprecipitation (ChIP) technique with chip technique (ChIP-on-chip) was used to reveal the target gene of SALL4 factor as a whole. The expression level of leukemic cells after knockout of SALL4 gene has been significantly changed, indicating that SALL4 is the key to regulate apoptosis. In addition, SALL4 is also associated with the progression of chronic myeloid leukemia (CML), which plays a key role in the survival and proliferation of CML tumor cells. Therefore, down-regulation of SALL4 level leads to cell cycle arrest and apoptosis of CML cells. ${ }^{[1]}$ It has been found that HOXA9 is one of the downstream target genes of SALL4 in AML, ${ }^{[12]}$ and SALL4 is located in the promoter region of the HOXA9 gene. Therefore, down-regulation of SALL4 will reduce HOXA9 expression and induce apoptosis. Kobayashi et al. ${ }^{[13]}$ suggested that knockout of SALL4 gene could arrest the cell cycle and induce cell growth inhibition in lung cancer. At the same time, the researcher also found that siRNA could be used to down-regulate SALL4 expression and arrest cell cycle in breast cancer to inhibit the proliferation of breast cancer cells. ${ }^{[14]}$ Chao HJ et al. ${ }^{[15]}$ suggested that knockout of SALL4 gene could inhibit the growth of endometrial carcinoma and carcinogenicity of tumors, inhibit cell proliferation and increase cell apoptosis.

\subsection{SALL4 and invasion \& metastasis}

Studies show that ${ }^{[16]}$ SALL4 gene expression level is closely related to lymph node metastasis of gastric cancer. Increasing of the expression of SALL4 leads to metastasis of human gastric cancer cells, but knockout of SALL4 via siRNA has the opposite effect. The possible mechanism is that SALL4 can strengthen the expression of $\mathrm{N}$-cadherin. At the same time, down-regulation of the expression of E-cadherin can induce epithelial-mesenchymal transition (EMT). Chao HJ et al. ${ }^{[15]}$ suggested that in endometrial carcinoma, SALL4 could specifically bind to the promoter of c-myc gene, and the reduction of SALL4 would reduce the expression of cmyc gene. It suggests that c-myc is the downstream target gene of endometrial oncogene and down-regulation of SALL4 can significantly reduce the metastasis and invasion of tumor cells. Itou et al. ${ }^{[17]}$ found that SALL4 factor inhibited the expression and transcription of E-cadherin in basal cell-like breast cancer cells. Kobayashi et al. ${ }^{[14]}$ showed that the expression of SALL4 was essential to the proliferation of cancer cells by siRNA test, and concluded that SALL4 was overexpressed in breast cancer tissues. 


\subsection{The role of SALL4 in epigenetics}

Epigenetic regulation of gene expression mainly includes epigenetic modification of four macromolecular mechanisms: DNA methylation, histone modification, chromatin remodeling and epigenetic modification of non-coding RNA. Yang et al. ${ }^{[18]}$ reported that SALL4 could interact directly with DNA methyltransferase, indicating that SALL4 gene can inhibit transcription by DNA methylation. In addition, Liu HX et al. ${ }^{[19]}$ demonstrated that SALL4 could bind target gene via the core protein complex 1 (PRC1). In addition, Liu et al. ${ }^{[20]}$ also found that SALL4 could inhibit stem cell gene transcription through methyltransferase 1 with histone lysine properties. Wang $\mathrm{F}$ et al. ${ }^{[21]}$ found that SALL4 could also activate HOXA9 gene expression together with histone methyltransferase. These results suggest that DNA mediated by SALL4 gene and methylation or demethylation of histone can significantly regulate the expression of oncogenes.

\subsection{SALL4 and tolerance}

The expression level of SALL4 gene is related to the effect of tumor therapy. There is a high expression of SALL4 gene in patients with AML resistance. Decreasing the expression of SALL4 gene in AML can increase the sensitivity of patients to drug therapy. Lin $\mathrm{BH}$ et al. ${ }^{[22]}$ have found that, ABCA3, called P-glycoprotein 3, is closely related to tumor drug resistance in hematological malignancies and multiple solid malignancies. Other scholars have confirmed that the expression of SALL4 gene is positively correlated with ABCA3. Tyrosine kinase inhibitor (TKI) upregulates the expression of $\mathrm{ABCA} 3$ and increases the expression of SALL4 gene in chronic myeloid leukemia cells. ${ }^{[23]}$ In addition, the expression of SALL4 gene will affect the sensitivity of endometrial cancer cells to carboplatin. ${ }^{[24]}$ Overexpression of SALL4 in the treatment of endometrial carcinoma with carboplatin will further increase the resistance of tumor cells to carboplatin. After treatment with carboplatin, the proliferation of endometrial carcinoma cells after transfection of SALL4 gene was significantly higher than that of the contrast group. At the same time, overexpression of SALL4 gene will protect endometrial cancer cells from apoptosis induced by carboplatin. Conversely, knockout of SALL4 gene in carboplatin-resistant endometrial cancer cells significantly enhances the efficacy of carboplatin

\section{References}

[1] Zang HJ. Global Cancer Report. Anticancer. 2015; 28(2):43-45.

[2] Rao S, Zhen S, Roumiantsev S, et al. Differential roles of Sall4 isoforms in embryonic stem cell pluripotency. Mol Cell Biol. 2010; 30(22): 5364-5380. PMid: 20837710. https://doi.org/10. 1128/MCB.00419-10 drugs. In addition, some scholars consider that overexpression of SALL4 gene will affect the survival rate and proliferation of liver cancer cells after 5-FU treatment of liver cancer. This suggests that the expression of SALL4 gene is also associated with the chemosensitivity of hepatocellular carcinoma cells. $^{[25]}$

\subsection{SALL4 gene and tumor markers and their clin- ical significance}

The expression of SALL4 gene has been reported as a sensitive marker in many malignant tumors, ${ }^{[26]}$ for example, acute B cell leukemia/lymphoma (B-ALL), myelodysplastic syndrome, breast cancer, endometrial carcinoma, germ cell tumor, liver cancer, gastrointestinal cancer, etc. The expression of SALL4 in human AML is related to the progress of disease and therapeutic efficacy. SALL4 can be used as a marker of diagnosis and prognosis of AML. Overexpression of SALL4 often leads to the shorter survival of the patients with liver cancer, and is an independent prognostic factor for the survival and recurrence of HCC patients. Cao et al. ${ }^{[27]}$ found that the positive rate of SALL4 sensitive index in germ cell tumor was $100 \%$. In non-germ cell tumors, however, there was little expression of SALL4 in both metastatic and primary tumors. This undoubtedly provides a good diagnostic reference for the differential diagnosis of germ cell malignant tumor. These studies suggest that high-level expression of SALL4 is a novel tumor marker for prognosis in cancer patients.

\section{Summary and prospect}

In present, SALL4 is with carcinogenesis, and can be detected with high sensitivity and specificity. Its mechanism of carcinogenesis and role and progress in human malignant tumors are worthy of further study and exploration. Further research and analysis may provide new methods and ideas for individualized treatment of malignant tumors and provide better theoretical basis and reference value for clinical application in the future.

\section{Conflicts of Interest Disclosure}

The authors have no conflicts of interest related to this article.

[3] Zhang X, Yuan X, Zhu W, et al. SALL4: An emerging cancer biomarker and target. Cancer Letters. 2014; 357(1): 5562. PMid: 25444934. https://doi.org/10.1016/j.canlet. 2014.11.037

[4] Choudhry Z, Rikani AA, Choudhry AM, et al. Sonic hedgehog signaling pathway: a complex network. Annu Rev Neurosci. 2014; 21(1): 28-31.

[5] Lin J, Ji RB, Qian J. Research Progress of Transcription Factor Boro 
Double Tree like Gene 4 (SALL4). Chinese Journal of Experimental Hematology. 2011; 19(3): 820-823. PMid: 21729579.

[6] Yang J, Gao C, Chai L, et al. A novel SALL4/OCT4 transcriptional feedback network for pluripotency of embryonic stem cells. PLoS One. 2010; 5(5): el0766. PMid: 20505821. https://doi.org/ 10.1371/journal.pone.0010766

[7] Gao C, Kong NR, Chai L. The role of stem cell factor SALL4 in leukemogenesis. Crit Rev Oncogenesis. 2011; 16(1/2): 117-127. PMid: 22150312. https://doi.org/10.1615/CritRev0ncog. v16.i1-2.110

[8] Ma Y, Cui W, Yang J, et al. SALL4, a novel oncogene, is constitutively expressed in human acute myeloid leukemia (AML) and induces AML in transgenic mice. Blood. 2006; 108(8): 2726-2735. PMid: 16763212. https://doi.org/10. 1182/blood-2006-02-001594

[9] Zhang WP, Yao HP, Tong XM. SALL4/BMI-1 Gene Expression in Myeloma and Its Clinical Significance. Chinese Journal of Health Inspection. 2011; 21(2): 290-292.

[10] Yu YC, Chen L. The Study of SALL4 Gene in Malignant Hematologic Diseases. International Journal of Oncology. 2011; 38(1): 64-66.

[11] Lu J, Ma Y, Kong N, et al. Dissecting the role of SALL4, a newly identified stem cell factor, in chronic myelognous leukemia. Leukemia. 2011; 25(7): 1211-1213. PMid: 21468036. https: //doi.org/10.1038/leu.2011.65

[12] Li A, Yang Y, Gao C, et al. A SALL4/MLL/HOXA9 pathway in murine and human myeloid leukemogenesis. J Clin Invest. 2013; 123(10): 4195-207. PMid: 24051379. https://doi.org/10. $1172 /$ JCI62891

[13] Kobayashi D, Kuribayashi K, Tanaka M, et al. Overexpression of SALL4 in lung cancer and its importance in cell proliferation. Oncol Rep. 2011; 26(4): 965-970. PMid: 21725617.

[14] Kobayashi D, Kuribayshi K, Tanaka M, et al. SALL4 is essential for cancer cell proliferation and is overexpressed at early clinical stages in breast cancer. Int J Oncol. 2011; 38(4): 933-939. PMid: 21274508.

[15] Chao HJ, Zhang M, Zhang YM, et al. The Study of Expression of SALL4 Gene in Cervical Carcinoma, Cervical Intraepithelial Neoplasia and Plasma. Journal of Nanjing Medical University (Natural Science Edition). 2014; 34(2): 188-191.

[16] Zhang $\mathrm{L}, \mathrm{Xu} \mathrm{Z}, \mathrm{Xu} \mathrm{X}$, et al. SALL4, a novel marker for human gastric carcinogenesis and metastasis. Oncogene. 2014; 33(48): 5491-5500. PMid: 24276240. https ://doi.org/10.1038/onc. 2013.495
[17] Itou J, Matsumoto Y, Yoshikawa K, et al. Sal-like 4 (SALL4) suppresses CDH1 expression and maintains cell dispersion in basallike breast cancer. Febs Letters. 2013; 587(18): 3115-3121. PMid: 23954296. https://doi.org/10.1016/j.febslet.2013.07. 049

[18] Yang J, Corsello TR, Ma Y. Stem cell gene SALL4 suppresses transcription through recruitment of DNA methyltransferases. J Biol Chen. 2012; 287(3): 1996-2005. PMid: 22128185. https://doi. org/10.1074/jbc.M111.308734

[19] Liu HX, Bai YF, Wang W, et al. The Role of SALL4 in the Regulation of Multidrug Resistance in Small Cell Lung Cancer and Its Clinical Significance. China Journal of Pathology. 2014; 28(9): 604608.

[20] Liu L, Souto J, Liao W, et al. Histone lysine-specific demethylase 1 (LSD1) protein is involved in sal-like protein 4 (SALL4)-mediated transcriptional repression in hematopoietic stem cells. J Biol Chen. 2013; 288(48): 34719-34728. PMid: 24163373. https://doi. org/10.1074/jbc.M113.506568

[21] Wang P. Clinical Application and Mechanism of Stem Cell Factor SALL4 in the Occurrence \& Development and Prognosis Judgment of MDS. Beijing: Peking Union Medical College; 2015.

[22] Lin BH, Chen J, Lu JQ, et al. Role of Overexpression of ABCA3 in Nasopharyngeal Carcinoma Tumor Stem Cells in the Mechanism of Cisplatin Resistance. Medical Guide. 2015; 34(11): 1422-1428.

[23] Hupfeld T, Chapuy B, Schrader V, et al. Tyrosinekinase inhibition facilitates cooperation of transcription factor SALL4 and ABC transporter A3 towards intrinsic CML cell drug resistance. Brit J Haematol. 2013; 161(2): 204-213. PMid: 23432194. https : //doi.org/10.1111/bjh.12246

[24] Li A, Jiao Y, Yong KJ, et al. SALL4 is a new target in endometrial cancer. Oncogene. 2013; 31(1): 63-72.

[25] Oikawa T, Kamiya A, Zeniya M, et al. Sal-like protein 4 (SALL4), a stem cell biomarker in liver cancers. Hepatology. 2013; 57(4): 1469-1483. PMid: 23175232. https ://doi.org/10.1002/hep. 26159

[26] Bu JS, Li BY, Qin CL. et al. Research Progress of SALL4 in Diseases. Chinese and Foreign Medical Research. 2011; 9(24): 157159.

[27] Cao D, Li J, Guo CC, et al. SALL4 is a Novel diagnostic marker for testicular germ cell tumors. Am J Surg Pathol. 2009; 933(7): 1065-1077. PMid: 19390421. https://doi.org/10.1097/PAS. Ob013e3181a13eef 